\title{
Black SEParation Strategies in Colour REPRODUCTION
}

\author{
AGIC, D.; Strgar KuRECIC, M.; MANDiC, L. \& PAP, K.
}

Abstract: The goal of the research presented in this paper was to investigate different strategies for black colour separation in four-colour CMYK reproduction process. This work describes some extended behaviour in making of separations by applying achromatic substitution strategies. The results have shown that appropriate substitution can lead to higher black ink amount then theoretical, when using some output device profiles. Additional consequences of that can be recognized in IR spectral domain, where the black ink renders significantly lower reflection then by using process inks. This effect enables the possibility of producing some special purpose graphics and security graphics.

Key words: colour reproduction, tertiary colours, achromatic substitution, IR response
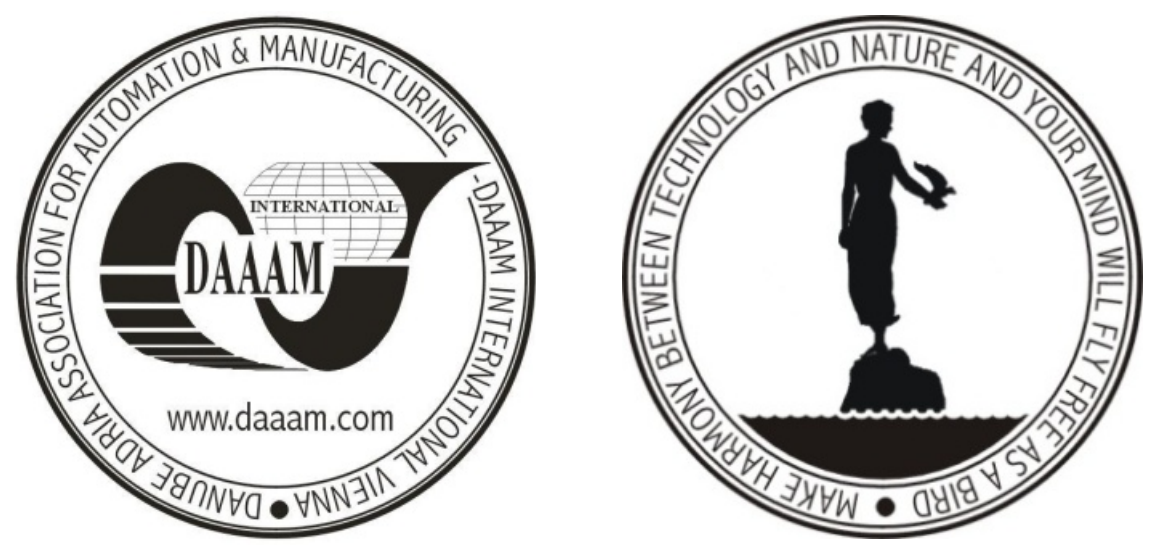

Authors' data: Univ.Prof. Agic, D[arko]; Dr. Sc. Strgar Kurecic, M[aja]; Dr. Sc. Mandic, L[idija]; Dr. Sc. Pap, K[laudio], University of Zagreb Faculty of Graphic Arts, Getaldiceva 2, 10000 Zagreb, Croatia, darko.agic@grf.hr, mstrgar@grf.hr, lidija.mandic@grf.hr

This Publication has to be referred as: Agic, D[arko]; Strgar Kurecic, M[aja]; Mandic, L[idija] \& Pap, K[laudio] (2009). Black Separation Strategies in Colour Reproduction, Chapter 01 in DAAAM International Scientific Book 2009, pp. 001008, B. Katalinic (Ed.), Published by DAAAM International, ISBN 978-3-90150969-8, ISSN 1726-9687, Vienna, Austria

DOI: $10.2507 /$ daaam.scibook.2009.01 